\title{
Pharmacogenomic biomarkers
}

\author{
Sandra C. Kirkwood* and Richard D. Hockett, Jr. \\ Eli Lilly and Company, Lilly Corporate Center, Indianapolis, IN 46285, USA
}

\begin{abstract}
Pharmacogenomic biomarkers hold great promise for the future of medicine and have been touted as a means to personalize prescriptions. Genetic biomarkers for disease susceptibility including both Mendelian and complex disease promise to result in improved understanding of the pathophysiology of disease, identification of new potential therapeutic targets, and improved molecular classification of disease. However essential to fulfilling the promise of individualized therapeutic intervention is the identification of drug activity biomarkers that stratify individuals based on likely response to a particular therapeutic, both positive response, efficacy, and negative response, development of side effect or toxicity. Prior to the widespread clinical application of a genetic biomarker multiple scientific studies must be completed to identify the genetic variants and delineate their functional significance in the pathophysiology of a carefully defined phenotype. The applicability of the genetic biomarker in the human population must then be verified through both retrospective studies utilizing stored or clinical trial samples, and through clinical trials prospectively stratifying patients based on the biomarker. The risk conferred by the polymorphism and the applicability in the general population must be clearly understood. Thus, the development and widespread application of a pharmacogenomic biomarker is an involved process and for most disease states we are just at the beginning of the journey towards individualized therapy and improved clinical outcome.
\end{abstract}

Keywords: Pharmacogenomics, biomarkers

\section{Introduction}

Recently, the sequence of the human genome was reported by two different groups [28,31]. This impressive scientific achievement has led to speculation about how this knowledge will profoundly change how we practice medicine and develop drugs. The ability to identify genes associated with the development of disease or those that can predict response to therapy or the development of a side effect has been touted as a means to improve therapeutic outcome [8]. The term "the right drug into the right patient" has often been used to describe the effects the genetic revolution will have on drug development. While studying the human genetic code will uncover secrets which will ultimately impact drug discovery and development, the evolution of genetic associations into genetic biomarkers requires multiple, time consuming steps. We are at the begin-

*Address for correspondence: Sandra C. Kirkwood, Eli Lilly and Company, Lilly Corporate Center DC 0444, Indianapolis, IN 46285, USA. Tel:: +1 317433 9492; Fax: +1 317277 2934; E-mail: skirkwood@lilly.com. ning of a long pursuit whose ultimate goal is improved patient care. However, for most disease states, genetic biomarkers to identify patients at risk for that disease, stratify patients by clinical outcome, indicate treatment response, or predict adverse event occurrences are in reality, several years away.

\section{Development of a genetic biomarker}

The development of any successful biomarker requires the completion of multiple steps including laboratory and clinical studies. The development of a genomic biomarker to stratify patients by susceptibility to disease or likely response to therapy, including drug efficacy or vulnerability to adverse event is no different (Fig. 1, Steps A, B, C). In addition to a complete understanding of the risk conferred by the genomic biomarker, a validated assay, with defined sensitivity and specificity, must be available. If the biomarker is intended to drive prescribing practices, regulatory approval will also be mandatory. 


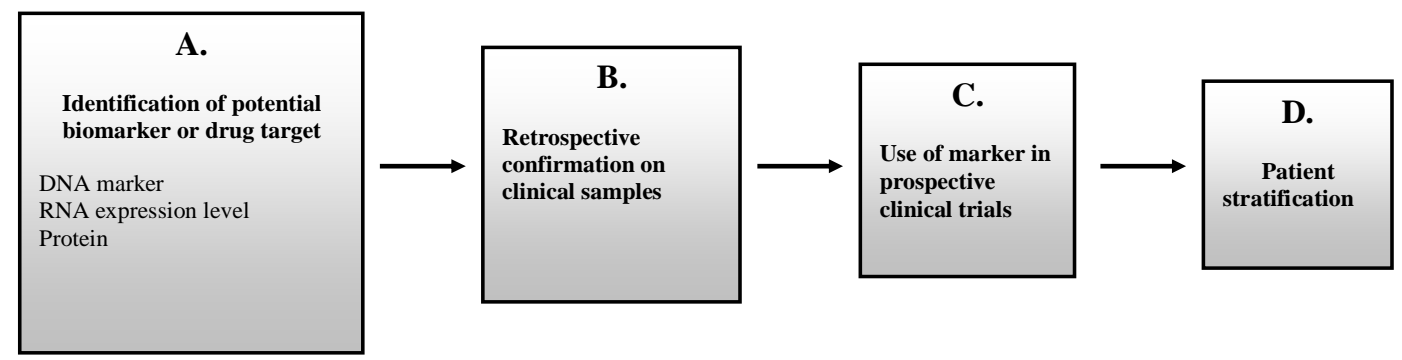

Fig. 1. Schematic development of a genetic biomarker. A) A biomarker is identified and associated with the phenotype in the laboratory. B) Once the genetic marker has been associated with the phenotype in the laboratory, the association is confirmed in samples from patients who have the phenotype or trait of interest. This may be stored samples or samples from a clinical trial not prospectively stratified by the biomarker. C) If part B is successful, then the marker is used prospectively in additional clinical trials to validate its use. D) The ultimate goal of this approach is to stratify patients by the biomarker, into groups by response to drug or susceptibility to side effect development.

The first step in the process is the identification and validation of the biomarker in a research laboratory (Fig. 1, Step A). The markers relationship with biologic function must be thoroughly tested and reproduced. A variety of methodologies are utilized in this phase to delineate the relationship. The objective is to frame a hypothesis that can be tested and validated in a clinical research setting clearly associating the biomarker with the phenotype of interest.

The next step, retrospective analysis, is the definitive association of the biomarker with the phenotype, drug effect or disease susceptibility in a sample of patients where the biomarker is not a priori used in their clinical management (Fig. 1, Step B). This may be achieved by an after the fact association of the biomarker to the outcome in a newly collected clinical trial sample or, alternatively, on a cohort of stored clinical samples with the appropriate phenotype. Both methods will be referred to herein as retrospective analyses. These retrospective studies provide the initial clinical evidence confirming the laboratory research and validating the hypothesis. This confirmation is essential to justify the next step, prospective clinical trials utilizing the biomarker in the evaluation or clinical management of the patient (Fig. 1, Step C).

The inability to validate at this stage and thus the demise of many biomarkers is often because of a poorly defined hypothesis, weak scientific evidence inadequately linking the phenotype and biomarker, or statistical issues such as deficiencies in study design and power. Furthermore, depending on the strength of the scientific evidence supporting the association, the risk attributable to the biomarker, and the general applicability of the marker in a variety of populations, multiple clinical association studies may be required prior to either regulatory approval or general acceptance by the medical community. The final step is the incorpo- ration of the biomarker into general medical practice and the prospective stratification of patients into groups based upon the biomarker (Fig. 1, Step D). Because of the requirement for regulatory approval for this final phase, realistic time expectations are measured in years to progress from A to D.

\section{Categorization of pharmacogenomic biomarkers}

For purposes of this manuscript genomic biomarkers are stratified into two main categories, disease susceptibility biomarkers and drug activity biomarkers (Table 1).

\subsection{Disease susceptibility biomarkers}

Genetic biomarkers for disease susceptibility include those for Mendelian and complex disease including cancer. Utilization of these biomarkers promises to result in improved understanding of the pathophysiology of disease, identify new potential therapeutic targets, and improve the molecular classification of disease.

\subsubsection{Mendelian disease}

For years biomarkers have been used to test for single gene mutations predisposing to disease. Until recently, the primary biomarkers were assays detecting the abnormal protein product of the mutated gene such as measuring phenylalanine levels while testing for phenylketonuria [9] and the sickledex test [4] for sickle cell anemia. With the advent of modern molecular biology, disease genes are now often identified using tests for the specific genes themselves. Examples of these include the trinucleotide $(\mathrm{CAG})$ repeat expansion in the Huntington's disease gene [22], the fibrillin-1 
Table 1

Categorization of pharmacogenomic biomarkers
I Disease Susceptibility Genes
A Mendelian Disease Genes
i Cystic Fibrosis
ii Hemophilia
iii Marfan Syndrome
B Complex Diseases
i Alzheimer's Disease
ii Coronary Artery Disease
iii Diabetes
C Genetic Changes of Tumorogenesis

II Drug Activity Biomarkers

A Genetic Polymorphisms Predicting Drug Metabolism

i $\mathrm{N}$-acetyl transferases

ii Cytochrome P450 genes

B Genetic Polymorphisms Predicting Drug Response

i Beta-2 adrenergic and asthma

ii 5-lipoxygenase and asthma

C Genetic Polymorphisms Predicting Adverse Events

gene in Marfanfls syndrome [12] and the identification of the mutations for cystic fibrosis, most frequently the delta F508 variant $[11,20,38]$. Even though the penetrance of these disease genes is often high with the presence of the disease gene conferring a high likelihood of manifesting the disease, the scientific validation of the identified mutations as the causative alleles can be arduous. However, once confirmed the clinical validation of these biomarkers is relatively straightforward, applying the same principles as any diagnostic test. Hundreds of commercial diagnostics for single gene disorders are available [18]. While knowledge of disease causing genes often illuminates the underlying biology and pathophysiology of disease identifying new potential targets, few to date have yielded curative therapeutics. The difficulty lies in relating the specific gene mutation to a "druggable" target.

\subsubsection{Complex disease genetics}

Complex human disorders, caused by multiple genetic and environmental factors, are characterized by high population prevalence, lack of clear Mendelian patterns of transmission, etiologic and phenotypic heterogeneity, and a continuum between disease and nondisease states [30,32]. The identification of genes influencing complex traits such as heart disease, diabetes, and cancer will not only assist in predicting those individuals who are predisposed to disease, but potentially have significant impact in the pharmaceutical industry by providing new targets for therapeutics. Currently all pharmaceutical products are aimed at approximately 500 targets. The human genome is estimated to consist of 30,000-60,000 genes, providing 60-120 times that number of potential targets. Either directly, or indirectly through protein-protein interactions or as part of feedback loops, the genes associated with complex diseases represent a significant pool of possible new targets. The use of novel genetic polymorphisms in the drug discovery process is a long road progressing through multiple phases similar to those previously outlined (Fig. 1).

The definitive association of the genetic polymorphism with disease phenotype is complicated by multiple factors including the limited understanding of the role these genetic factors play in the pathophysiology of disease and the influence of multiple genetic and environmental co-factors on the disease. Essential to the successful pursuit of complex disease genes is accurate phenotypic classification. If the clinical phenotyping is inaccurate the association of a genetic variant with that phenotype is complicated by false positive and false negative associations. Thus, a major challenge facing the development of genetic biomarkers in complex disease is etiologic or phenotypic heterogeneity. Heterogeneity not only influences the ability to discover a biomarker but also the clinical utility of the biomarker once identified. Clearly, a biomarker that is specifically associated with the phenotype of interest has more clinical utility than one associated with a range of phenotypes [30]. To counter this inherent difficulty and aid in the discovery of applicable genetic biomarkers, genetic epidemiology principles are being applied to refining phenotypic definitions and identify the role of genetic and environmental risk factors in disease and drug response.

The linkage and association of CAPN10, the gene encoding the cysteine protease calpain-10, with type II diabetes, is a recent example of successfully identifying a genetic variant associated with a complex disease [21]. Initially, the inheritance of specific CAPN10 haplotypes was associated with an increased risk for type II diabetes in Mexican Americans from a single county in Texas. Replication in two northern European populations, a population from the Botnia region of Finland and the German population of Saxony, provided confirmation and evidence for the general applicability of the CAPN10 association. However, the role of CAPN10 in type II diabetes and how a ubiquitous serine protease affects glucose control, the effect of the polymorphism 
in the calpain-10 locus on the protein product, or how this might relate to the development of type II diabetes is unclear [3]. This uncertainty regarding the role of calpain-10 illustrates one of the complicating factors in developing biomarkers for complex traits, the difficulty in understanding the role these genetic factors play in the pathophysiology of disease. Following association of a genetic variant with disease, studies demonstrating a functional change related to the genotype, not simply an association secondary to the polymorphism being in disequilibrium with the causative gene, are necessary.

Following an understanding of the relationship between the identified biomarker and the phenotype, all additional polymorphisms in the gene or its regulatory region and their relationship to the observed functional effect should be investigated. This phase of investigation often includes studies in a variety of populations, as a specific polymorphism may have utility in one but not in another. To continue with the diabetes examples, this is an active area of investigation for CAPN10. In a population of Caucasians of British/Irish ancestry, Evans et al. [16] were unable to replicate the association of the specific CAPN10 alleles previously identified but rather associated additional alleles at the locus with increased type II diabetes risk. The inability to replicate and the differing polymorphisms and haplotypes associated between populations may be due to multiple susceptibility alleles at CAPN10 or different patterns of linkage disequilibrium between a common causal variant [16]. This illustrates the necessity of large population-based association studies to better understand both the strength and specificity of the association of CAPN10 polymorphisms to type II diabetes risk. Only after a more complete understanding of the polymorphisms' relationship to diabetes and the protease's function can the gene be investigated as a new therapeutic target. The steps in this process are not trivial and the development of viable pharmaceuticals will likely take $5-10$ years without substantial luck by the investigators.

\subsubsection{Genetic changes associated with tumorogenesis}

By definition cancer is a genetic disease requiring a progression of genetic changes for tumor formation, the multi-hit theory of tumorogenesis [27,29]. Comparing the genetic background of the tumor with the genetic background of the patient is a powerful investigative tool often implicating genes involved in tumor formation. Although several small molecules aimed at inhibiting genes and proteins implicated in cancer formation are in development, most genetic changes in the cascade are not utilized as specific therapeutic targets. Herceptin $^{\mathrm{TM}}$ and Gleevec ${ }^{\mathrm{TM}}$, two notable exceptions, are therapeutics designed directed at a genetic target. Gleevec $^{\mathrm{TM}}$ for the treatment of chronic myelogenous leukemia (CML) is specifically designed at a genetic target, the bcr-abl fusion gene or the Philadelphia chromosome. The bcr-abl fusion gene results from a reciprocal translocation between chromosome 9 and 22 and the gene product demonstrates increased kinase activity. Bcr-abl is an attractive target as it is sufficient to cause disease and is present in over $95 \%$ of patients with CML [23]. Gleevec ${ }^{\mathrm{TM}}$ is a tyrosine kinase inhibitor designed to block the ability of bcr-abl to phosphorylate its unknown substrate [24].

In general medical practice the most widespread use of genetic biomarkers to identify patients eligible for treatment is in the identification of breast cancer patients eligible for treatment with Herceptin. HER2 is the protein product of the oncogene erbB-2, a member of the growth factor family of receptors [39]. In approximately one-third of breast cancer cases, the overexpression of HER2 on the surface, closely linked to the amplification of the erbB-2 DNA, contributes to the growth characteristics of the tumor. Genentech developed Herceptin, a humanized trastuzumab antibody against HER2, for the treatment of HER2 overexpressing breast cancer [7]. Treatment with Herceptin in HER2 overexpressing breast cancers especially in combination with other chemotherapeutic agents is now considered the standard of care. Since breast cancer with normal levels of HER2 expression does not respond to Herceptin, an assay to identify patients eligible for treatment with Herceptin is rational; thus, immunohistochemistry (IHC) and fluorescence in situ hybridization (FISH) assays were developed [5,19,25].

The development of Gleevec and Herceptin illustrates the complexity of developing a directed therapeutic, aimed at a genetic target $[5,14,19,25]$. The process included identification of an appropriate target, testing the compound in a number of preclinical models, conducting human ex-vivo studies, ultimately culminating in phase I, II and III clinical trials and FDA approval. In order to proceed to generalized use, and convince the appropriate regulatory agencies, the science supporting the rationale as well as the methodology must be sufficiently compelling. This usually also means the development of a diagnostic assay and more than one study confirming the sensitivity and specificity. Multiple studies generally require multiple years. Therefore, the claim that we will have an explosion of genetic markers suitable for general use in the immediate future appears unrealistic. 
A potentially more timely clinical utility of gene expression changes with tumor formation will be in the reclassification of tumors. Tumor classification currently relies upon morphology, tumor size, and at times cell surface protein expression. These parameters are widely used to help determine prognosis, but have not been helpful in identifying those likely to respond or not to various therapeutics and personalizing prescriptions. A better understanding of the molecular basis of tumors and a more comprehensive assessment of expressed genes may enable the identification of those patients likely to respond to particular treatments, in addition to identifying new targets for directed therapeutics such as Gleevec and Herceptin.

The most inclusive technique for gene expression analysis is array profiling, which monitors the RNA expression of multiple genes simultaneously. Recent reports have used array profiling in the classification of B cell lymphomas [2,26], epithelial ovarian cancer [1], and breast cancer [35]. In these reports, total RNA from each tumor was hybridized to gene chips with thousands of human sequences and the tumors classified based on the expression profile. As with any novel technique, the disadvantage for array profiling is the newness of the technology. Prior to widespread utility in a clinical setting, a complete understanding of the capabilities, limitations, and reproducibility of these techniques is needed. Until the variability and inherent noise level are understood, the robustness of the technology and the ability to detect minor changes in expression level remain uncertain. While the technology seems adept when comparing groups of individuals, the utility for an individual patient remains unknown. Array technology has not been utilized in prospective clinical trials under regulatory scrutiny, and until it is, the utility of microarray technology in clinical trials is difficult to determine.

\subsection{Drug activity biomarkers}

The primary focus in the pharmaceutical industry is on the utilization of genetics to develop rational therapeutics. As described above, the identification of genetic biomarkers for disease susceptibility may lead to new targets and better classification of disease. However essential to more personalized prescribing practices is the identification of biomarkers that stratify individuals into groups based on likely response to a particular therapeutic, both efficacy and likelihood of experiencing an undesired reaction.

\subsubsection{Genetic polymorphisms predicting drug metabolism}

The most common application of genetic markers in drug development and medicine today is the genotyping of the drug metabolism enzymes. Drug metabolizing enzymes with known genetic polymorphisms include the cytochrome $\mathrm{P} 450$ enzymes such as CYP2D6, CYP3A1, CYP2C9, CYP2C19, CYP2E1, and CYP2A6 and the glutathione S-transferases and $\mathrm{N}$-acetyl transferases [37]. The list of drugs metabolized by each enzyme system is large, and reviewed elsewhere $[6,36]$. Unlike disease-associated variants, the genetic polymorphisms in these enzymes do not predict disease or response to therapy but lead to differences between individuals in metabolic activity and clearance of drugs. Approximately $25 \%$ of the current commercially available drugs [34] are metabolized by one of the cytochrome P450 enzymes, CYP2D6. CYP2D6 has at least 37 known polymorphisms, some exhibiting decreased or absent enzyme activity [10]. An individual with two copies of a slow metabolizing variant has significantly reduced clearance of the parent drug, resulting in a prolonged half-life and potentially warranting a reduced dose. The drug metabolizing enzymes are not targets for new therapeutics but rather may require dosing regimen alterations for therapeutics metabolized by the enzyme. Even though these tests are not widely used outside of clinical trials, there is growing acceptance for their use in determining proper dosing regimens.

\subsubsection{Genetic polymorphisms predicting drug response}

In addition to polymorphisms in the genes encoding drug-metabolizing enzymes affecting dosing regimens, an individual's clinical response to treatment may be influenced by genetic variants in the coding or regulatory regions of genes involved in the therapeutic pathways. Therefore, genetic biomarkers for response may facilitate classification of individuals by response, improving therapeutic outcome, and personalizing prescriptions. Although the clinical utility of such genetic biomarkers has not yet been established, recently a few publications have reported the first step, the association of genetic variants with clinical drug response.

The response to $\beta$ agonists aimed at reversing the acute bronchospasm in asthma has been associated with polymorphisms in the $\mathrm{B}_{2}$ adrenergic gene. The genetic polymorphisms identified in $\mathrm{B}_{2}$ adrenergic receptor gene and associated with response to $\beta$ agonists have also been shown to have functional consequences in 
vitro and in vivo. However, conflicting results have been reported and the relationship between the genetic variants and bronchodilatory response to $\beta$ agonists remains unclear. As outlined for complex trait association studies, the analysis is complex and a multitude of reasons could account for the conflicting results. One possibility is that a haplotype, or combination of single nucleotide polymorphisms, rather than an individual variant is associated with clinical response. For example, a recent report utilized phylogenetic analysis to organize thirteen single nucleotide polymorphisms into twelve estimated haplotypes in a sample of Caucasians. The analysis associated some of these haplotypes but not any of the individual polymorphisms with bronchodilatory response to albuterol [15]. The biological effect, differential response, may be due to interactions involving transcription, translation, and protein processing of the multiple polymorphisms comprising the haplotype. As illustrated with CAPN10, the association of an estimated haplotype in a relatively small population is just the initial step in a long process. The association of the $\mathrm{B}_{2}$ adrenergic receptor gene needs to be replicated in a larger sample and the functional consequences of the polymorphisms understood in a variety of populations.

Another genetic variant associated with the clinical response to drug treatment recently reported in the literature is the 5-lipoxygenase (ALOX5) promoter genotype's association with response to anti-asthma treatment [13]. Tissue culture experiments associated DNA sequence variants in the regulatory region of ALOX5 with diminished promoter activity. Analyzing the results from a clinical trial for ABT-761, a selective inhibitor of ALOX5, demonstrated that individuals homozygous for rare variants demonstrated significantly decreased response as measured by FEV1 when compared with individuals heterozygous and homozygous for the wild-type alleles. Although individuals homozygous for the rare variants appear to explain a portion of the individuals who do not respond to ALOX5 inhibition, a biomarker for the ALOX5 variants is not yet ready for routine use. The report by Drazen included only 10 individuals homozygous for the mutant allele [13]. Prior to implementation of routine genetic testing of the ALOX5 promoter in the clinic, the results of this study need to be replicated in a larger trial with multiple outcome measures. In addition, as approximately only $6 \%$ of non-responders carry the allele at the ALOX5 promoter locus, there may be other genetic defects in the pathway yet to be identified. Identification of genetic biomarkers for drug response has potential applicability in understanding those likely to respond to a particular therapeutic. However, as these recent reports in the literature illustrate, these markers are in the early stages of development.

\subsubsection{Predicting adverse events or side effects}

Adverse events related to drug therapy are not uncommon. Fortunately, most are mild and do not require cessation of therapy. However more serious adverse events result in significant morbidity and mortality. Drugs fail during development and in several instances even after launch due to the occurrence of adverse events. Often touted as one area of significant potential impact for pharmacogenomics, prediction of those patients likely to experience a drug induced adverse event would greatly benefit pharmaceutical companies and medicine in general. Unfortunately, aside from those genetic differences predicting altered drug metabolism and the associated drug toxicity, no examples exist where pharmacogenomics has predicted these unwanted occurrences.

Nonetheless, genetics plays a large role in patients' reactions to medications, and pharmacogenomics offers great potential to avert unwanted side effects. For a genetic biomarker to have clinical utility, we propose that at least four criteria are needed: 1) the adverse event must be relatively frequent, 2) the adverse event must be non-life threatening, 3) the adverse event must be less or equal in severity to the medical condition requiring treatment, and 4) the therapy must fill a niche in the marketplace such as few alternatives for therapy are available or a compelling reason to take the medication exists, such as a better formulation or improved efficacy. These conditions are especially true for the first drug to place genetic testing for susceptibility to adverse event in its label.

A recent example of a drug taken off the market following adverse event occurrence, troglitazone, will be used to highlight the reasoning behind these criteria. During the clinical development of troglitazone, a thiazolidinedione antidiabetic agent for the management of type II diabetes mellitus, some degree of liver toxicity was noted in 48 of approximately 2500 patients, 20 of which withdrew from treatment [17]. Following discontinuation of therapy, the elevation of liver enzymes reversed and the FDA subsequently licensed the drug. During the first two years after launch, $\sim 1,000,000$ patients were placed on troglitazone with 70 experiencing liver failure, 60 deaths and 10 transplants, which lead to troglitazone's recall [17]. 
The troglitazone example helps to illustrate the rationale behind these criteria. To power the analysis to definitively associate a genetic biomarker with the adverse event a sufficient number of patients need to experience the event. In the troglitazone example, 70 out of $\sim 1,000,000$ patients had severe liver toxicity. This is likely not sufficient to power an association study even if a single genetic polymorphism confers a high risk. Liver toxicity associated with troglitazone use is likely multifactorial with multiple genetic and environmental factors of moderate effect involved. Assuming that DNA is available from all of the 70 individuals experiencing the adverse event and this sufficiently powers the study for the initial association, an additional $\sim 1,000,000$ patients must evaluated to get the confirmatory sample of 70 individuals. The ethical and legal issues involved in collecting the replicate samples, the safety of these individuals, and the cost make it unlikely that any pharmaceutical company would support this effort.

Due to the rigorous monitoring of drug related adverse events, with serious side effects, the recall process is fortunately invoked long before sufficient numbers of patients exist to prove causation. Further limiting the sample size is the availability of DNA from the individuals who experienced the adverse event. In the event of death, DNA may not be available from the individuals who experienced the event. In addition, those who experience serious adverse events or their survivors may be unwilling to cooperate with the drug manufacturer. Following the disclosure of the side effects related to troglitazone therapy and the availability of alternative treatments, physicians and patients would be unlikely to take the risk and agree to troglitazone treatment. In the case of a less severe adverse event, a medical condition more acute severity, or where few or no alternative therapies are available, pharmacogenomics may have more applicability.

At present genetic biomarkers may have greater utility for adverse event prediction in the pre-clinical arena. Although animal models have been helpful in triaging drugs that are clearly toxic to the liver, kidney, and heart, these models do not accurately predict all toxicity seen in humans. If correlation between animal models and human outcomes can be established, especially at the transcriptional level, the effect of drugs under development in the appropriate animal model could dictate the future of the drug. A drug under development could be terminated sooner and failed human trials prevented if the correlation between clinical liver, kidney, or heart toxicity in humans and the pre-clinical models could be improved. While no examples are evident to date, the potential for preventing human morbidity is worth a considerable investment.

\section{Conclusion}

Genetic biomarkers hold great promise for the future of medicine. The identification of genetic markers of disease susceptibility, tumor classification, metabolism, dose response, and the development of adverse events has the potential to improve therapeutic outcome. However, prior to widespread clinical application of a genetic biomarker multiple scientific and clinical studies must be completed to identify the genetic variants and delineate their functional significance in the pathophysiology of a carefully defined phenotype. What information is conferred by the biomarker including the risk conferred by the polymorphism and the applicability in the general population must be clearly understood. Subsequently, a validated assay with understood specificity and sensitivity must be developed and available for general use.

In addition, prior to the general application of genetic markers in the clinical setting, issues related to privacy and the ethical and legal use of genetic data must be addressed [33]. The difficult genetic privacy concerns may be avoided in a scientific research setting as the research may proceed on anonymized samples, without identifiable links to the individual. However, prior to use in the general clinical setting, patients and practitioners need to be educated regarding the information gleaned from genetic biomarkers and the importance of environmental factors. The concerns regarding privacy and confidentiality, the potential for genetic discrimination, and the societal implications for increased information about human variation need to be addressed [33]. Genetics will provide valuable information to assist in the management of patients and has the potential to revolutionize medicine. However, the development and application of genetic biomarkers is an involved process and we are just at the beginning of a long journey toward improved therapeutic outcome.

\section{References}

[1] A.A. Alaiya, B. Franzen, A. Hagman, C. Silfversward, B. Moberger, S. Linder and G. Auer, Classification of human ovarian tumors using multivariate data analysis of polypeptide expression patterns, Int J Cancer 86(5) (1 June 2000), 731736. 
[2] A.A. Alizadeh, M.B. Eisen, R.E. Davis, C. Ma, I.S. Lossos, A. Rosenwald, J.C. Boldrick, H. Sabet, T. Tran, X. Yu, J.I. Powell, L. Yang, G.E. Marti, T. Moore, J. Jr. Hudson, L. Lu, D.B. Lewis, R. Tibshirani, G. Sherlock, W.C. Chan, T.C. Greiner, D.D. Weisenburger, J.O. Armitage, R. Warnke and L.M. Staudt et al., Distinct types of diffuse large B-cell lymphoma identified by gene expression profiling, Nature 403(6769) (3 Feb 2000), 503-511.

[3] L.J. Baier, P.A. Permana, X. Yang, R.E. Pratley, R.L. Hanson, G.Q. Shen, D. Mott, W.C. Knowler, N.J. Cox, Y. Horikawa, N. Oda, G.I. Bell and C. Bogardus, A calpain-10 gene polymorphism is associated with reduced muscle mRNA levels and insulin resistance, J Clin Invest Oct 106(7) (2000), R69-73.

[4] D.M. Canning and R.G. Huntsman, An assessment of Sickledex as an alternative to the sickling test, J Clin Pathol. 23(8) (Nov 1970), 736-737.

[5] W. Check, CAP Today 9 (14 Sep 2000)

[6] S. Cholerton, A.K. Daly and J.R. Idle, The role of individual human cytochromes P450 in drug metabolism and clinical response, Trends Pharmacol Sci. 13(12) (Dec 1992), 434-439.

[7] M.A. Cobleigh, C.L. Vogel and D. Tripathy et al., Multinational study of the efficacy and safety of humanized antiHER2 monoclonal antibody in women who have HER2overexpressing metastatic breast cancer that has progressed after chemotherapy for metastatic disease, J Clin Oncol 17 (1999), 2639-2648.

[8] F.S. Collins and V.A. McKusick, Implications of the Human Genome Project for Medical Science, JAMA 285(5) (2001), 540-544.

[9] G.C. Cunningham, Phenylketonuria testing - its role in pediatrics and public health, CRC Crit Rev Clin Lab Sci. 2(1) (Jan 1971), 45-101.

[10] A.K. Daly, J. Brockmoller, F. Broly, M. Eichelbaum, W.E. Evans, F.J. Gonzalez, J.D. Huang, J.R. Idle, M. IngelmanSundberg, T. Ishizaki, E. Jacqz-Aigrain, U.A. Meyer, D.W. Nebert, V.M. Steen, C.R. Wolf and U.M. Zanger, Nomenclature for human CYP2D6 alleles, Pharmacogenetics 6(3) (June 1996), 193-201.

[11] K. Davies, Genetic screening for cystic fibrosis, Nature 357(6377) (6 June 1992), 425.

[12] H.C. Dietz, G.R. Cutting, R.E. Pyeritz, C.L. Maslen, L.Y. Sakai, G.M. Corson, E.G. Puffenberger, A. Hamosh, E.J. Nanthakumar, S.M. Curristin, G. Stetten, D.A. Meyers and C.A. Francomano, Marfan syndrome caused by a recurrent de novo missense mutation in the fibrillin gene, Nature 352 (1991), 337-339.

[13] J.M. Drazen, C.N. Yandava, L. Dube, N. Szczerback, R. Hippensteel, A. Pillari, E. Israel, N. Schork, E.S. Silverman, D.A. Katz and J. Drajesk, Pharmacogenetic association between ALOX5 promoter genotype and the response to anti-asthma treatment, Nature Genetics 22(2) (June 1999), 168-170.

[14] B.J. Druker and N.B. Lydon, Lessons learned from the development of an abl tyrosine kinase inhibitor for chronic myelogenous leukemia, J Clin Invest. 105 (2000), 3-7.

[15] C.M. Drysdale, D.W. McGraw, C.B. Stack, J.C. Stephens, R.S. Judson, K. Nandabalan, K. Arnold, G. Ruano and S.B. Liggett, Complex promoter and coding region beta 2-adrenergic receptor haplotypes alter receptor expression and predict in vivo responsiveness, Proc Natl Acad Sci USA 97(19) (12 Sep 2000), 10483-10488.

[16] J.C. Evans, T.M. Frayling, P.G. Cassell, P.J. Saker, G.A. Hitman, M. Walker, J.C. Levy, S. O'Rahilly, P.V. Rao, A.J. Bennett, E.C. Jones, S. Menzel, P. Prestwich, N. Simecek, M. Wishart, R. Dhillon, C. Fletcher, A. Millward, A. Demaine, T.
Wilkin, Y. Horikawa, N.J. Cox, G.I. Bell, S. Ellard, M.I. McCarthy and A.T. Hattersley, Studies of association between the gene for calpain-10 and type 2 diabetes mellitus in the United Kingdom, Am J Hum Genet. 69(3) (Sep 2001), 544-552.

[17] E. Gale, Lessons from the glitazones: a story of drug development, Lancet 357 (2001), 1870-1875.

[18] GeneTests, http://www.genetests.org, National Institutes of Health, 2001.

[19] W. Hanna, Testing for HER2 Status, Oncology 61 (Oct 2001), S2:22-30.

[20] W.E. Highsmith Jr, G.L. Chong, H.T. Orr, T.R. Perry, D. Schald, R. Farber, K. Wagner, M.R. Knowles, W.J. Warwick and L.M. Silverman et al., Frequency of the delta Phe508 mutation and correlation with XV.2c/KM-19 haplotypes in an American population of cystic fibrosis patients: results of a collaborative study, Clin Chem 36(10) (Oct 1990), 1741-1746.

[21] Y. Horikawa, N. Oda, N.J. Cox, X. Li, M. Orho-Melander, M. Hara, Y. Hinokio, T. Linder, H. Mashima, P. Scwarz, L. del Bosque-Plata, Y. Horikawa, Y. Oda, I. Yoshiuchi, S. Colilla, K. Polonsky, S. Wei, P. Concannon, N. Iwaski, J. Schulze, L. Baier, C. Bogardus, L. Groop, E. Boerwinkle, C. Hanis and G. Bell, Genetic variation in the gene encoding calpain-10 is associated with type 2 diabetes mellitus, Nature Genet 29 (2000), 163-175.

[22] Huntington Disease Collaborative Research Group, A novel gene containing a trinucleotide repeat that is expanded and unstable on Huntington's disease chromosomes, Cell 72(6) (26 March 1993), 971-983.

[23] M. Kalidas, H. Kantarjian and M. Talpaz, Chronic myelogenous leukemia, JAMA 286(8) (22-29 Aug 2001), 895-898.

[24] H. Kantarjian, C. Sawyers, A. Hochhaus, F. Guilhot, C. Schiffer, C. Gambacorti-Passerini, D. Niederwieser, D. Resta, R. Capdeville, U. Zoellner, M. Talpaz, B. Druker and The International STI571 CML Study Group, Hematologic and cytogenetic responses to imatinib mesylate in chronic myelogenous leukemia, $N$ Engl J Med. 346(9) (28 Feb 2002), 645-652.

[25] S. Kaptain, L.K. Tan and B. Chen, Her-2/neu and breast cancer, Diagn Mol Pathol 10(3) (Sep 1990), 139-152.

[26] J. Khan, J.S. Wei, M. Ringner, L.H. Saal, M. Ladanyi, F. Westermann, F. Berthold, M. Schwab, C.R. Antonescu, C. Peterson and P.S. Meltzer, Classification and diagnostic prediction of cancers using gene expression profiling and artificial neural networks, Nat Med. 7(6) (June 2001), 673-679.

[27] K.W. Kinzler, M.C. Nilbert, L.K. Su, B. Vogelstein, T.M. Bryan, D.B. Levy, K.J. Smith, A.C. Preisinger, P. Hedge and D. McKechnie et al., Identification of FAP locus genes from chromosome 5q21, Science 253(5020) (9 Aug 1991), 661665.

[28] E.S. Lander, L.M. Linton, B. Birren and C. Nusbaum, Initial sequencing and analysis of the human genome, Nature 409(6822) (2001), 860-921.

[29] C. Lengauer, K.W. Kinzler and B. Vogelstein, Genetic instabilities in human cancers, Nature 396(6712) (17 Dec 1998), 643-649.

[30] K.R. Merikangas and J.D. Swendsen, Genetic Epidemiology of psychiatric disorders, Epidemiolo Rev 19 (1997), 144-155.

[31] No author, The human genome. Science genome map, Science 291(5507) (2001), 1218

[32] N. Risch and K. Merikangas, The future of genetics of complex diseases, Science 273 (1996), 1516-1517.

[33] M.A. Rothstein, Genetic Secrets: Protecting Privacy and confidentiality in the Genetic Era. Yale University Press. New Haven, Connecticut, 1997. 
[34] V.P. Shah, A. Yacobi, W.H. Barr, L.Z. Benet, D. Breimer, M.R. Dobrinska, L. Endrenyi, W. Fairweather, W. Gillespie, M.A. Gonzalez, J. Hooper, A. Jackson, L.J. Lesko, K.K. Midha, P.K. Noonan, R. Patnaik and R.L. Williams, Evaluation of orally administered highly variable drugs and drug formulations, Pharm Res. 13(11) (Nov 1996), 1590-1594.

[35] T. Sorlie, C.M. Perou, R. Tibshirani, T. Aas, S. Geisler, H. Johnsen, T. Hastie, M.B. Eisen, M. van de Rijn, S.S. Jeffrey, T. Thorsen, H. Quist, J.C. Matese, P.O. Brown, D. Botstein, P. Eystein Lonning and A.L. Borresen-Dale, Gene expression patterns of breast carcinomas distinguish tumor subclasses with clinical implications, Proc Natl Acad Sci USA 98(19) (11 Sep 2001), 10869-10874.
[36] M. Spatzenegger and W. Jaeger, Clinical importance of hepatic cytochrome P450 in drug metabolism, Drug Metab Rev. 27(3) (1995), 397-417.

[37] D.S. Streetman, J.S. Jr. Bertino and A.N. Nafziger, Phenotyping of drug-metabolizing enzymes in adults: a review of in-vivo cytochrome $\mathrm{P} 450$ phenotyping probes, Pharmacogenetics 10(3) (April 2000), 187-216.

[38] B.S. Wilfond, Screening policy for cystic fibrosis: the role of evidence, Hastings Cent Rep. 25(3) (May-Jun 1995), S21-23.

[39] C. Wright, K. Prasad and T.J. Lennard, ErbB2 expression in breast and other human tumors, J Clin Pathol. 45(5) (May 1992), 459-460. 


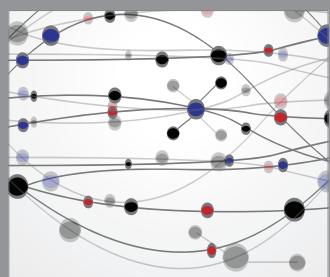

The Scientific World Journal
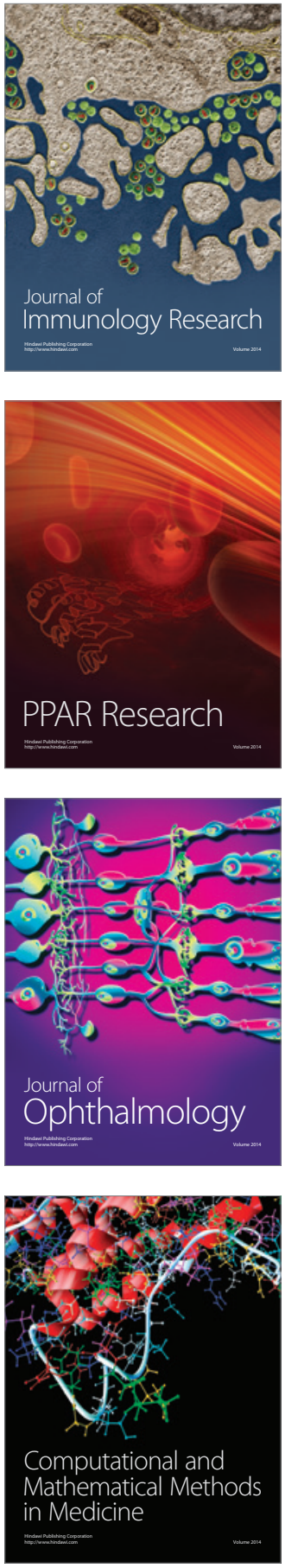

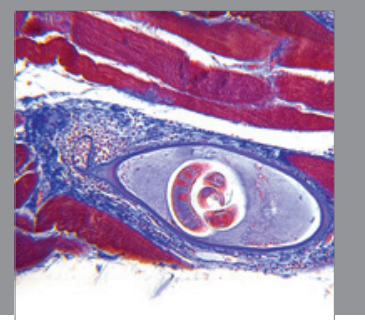

Gastroenterology

Research and Practice
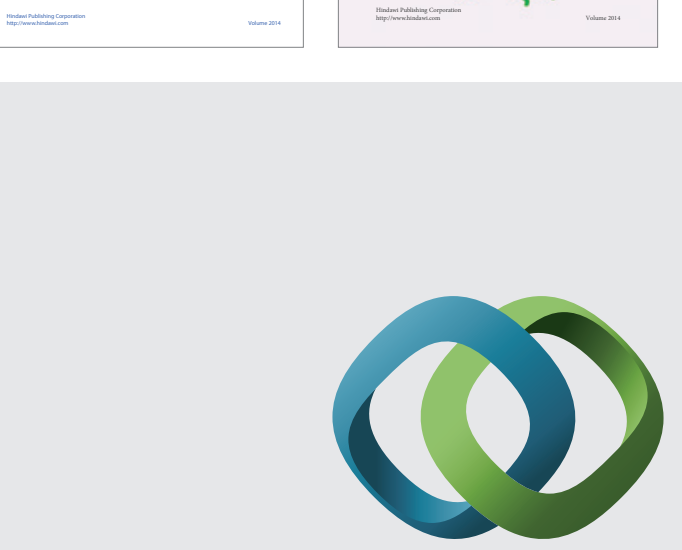

\section{Hindawi}

Submit your manuscripts at

http://www.hindawi.com
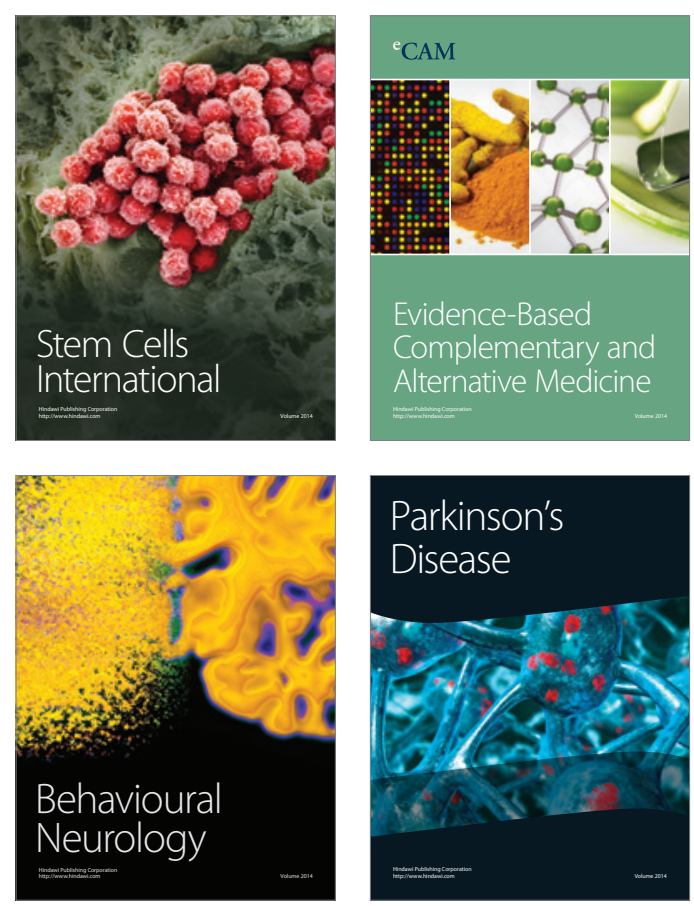

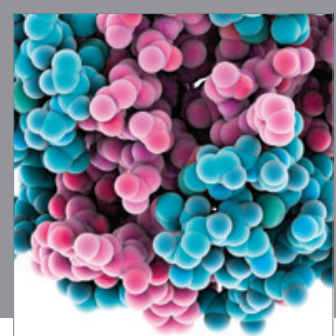

Journal of
Diabetes Research

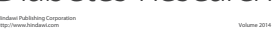

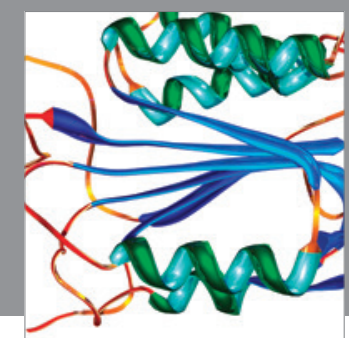

Disease Markers
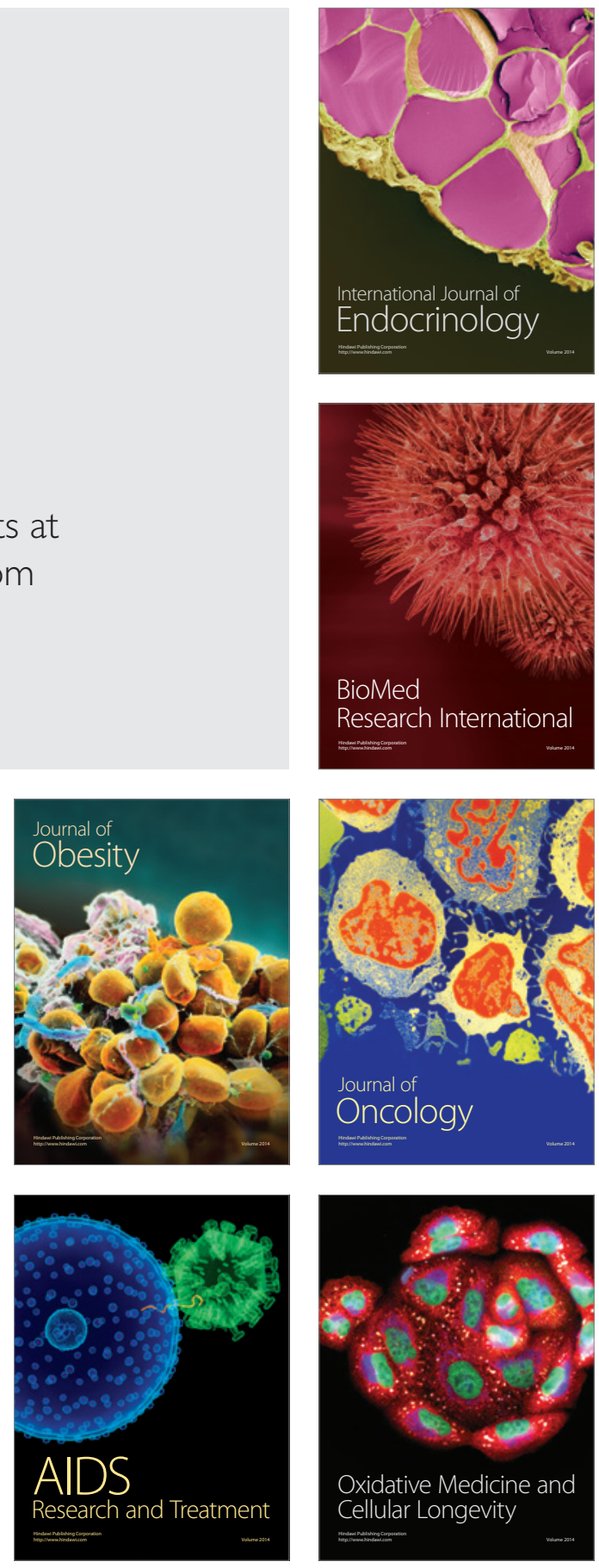